\title{
Role of Dual-Energy Computed Tomography in Characterization of Ureteric Calculi and Urinary Obstruction
}

Anchal Singh ${ }^{1}$, Sachin Khanduri ${ }^{2}$, Nazia Khan ${ }^{1}$, Poonam Yadav ${ }^{3}$, Mushahid Husain ${ }^{2}$, Ahmad Umar Khan ${ }^{1}$, Mazhar Khan ${ }^{4}$, Shreshtha Jain ${ }^{2}$

1. Radiodiagnosis, Era's Lucknow Medical College and Hospital, Lucknow, IND 2. Radiology, Era's Lucknow Medical College and Hospital, Lucknow, IND 3. Radiology, Era's Lucknow Medical College and Hospital, Lucknow, IND 4. Radiology, All India Institute of Medical Science, Patna, IND

Corresponding author: Sachin Khanduri, drsachinrad@gmail.com

\section{Abstract}

Objective

The present study was carried out to assess the accuracy of dual-energy computed tomography (DECT) in the morphological and chemical characterization of ureteric calculi along with the prediction of the grade of urinary obstruction.

\section{Methods}

This was a prospective observational study that included 100 cases with ultrasonography (USG)-diagnosed ureteric calculi that underwent surgery or had spontaneous expulsion of ureteric calculi. At enrolment, DECT was performed for an in vivo evaluation of volume, chemical composition, and grade of obstruction by subjective assessment of the perinephric edema. After surgical intervention, in vitro evaluation of volume was done by fluid displacement followed by infrared spectroscopy (IRS) for chemical composition. DECT findings were compared with the biochemical analysis and degree of obstruction was validated against excretory CT urograms. Sensitivity, specificity, and the positive predictive and negative predictive values of DECT were assessed.

\section{Results}

No significant difference was observed between the mean volume of stones by fluid displacement $\left(65.1 \pm 77.61 \mathrm{~mm}^{3}\right)$ and DECT assessment $\left(66.09 \pm 81.78 \mathrm{~mm}^{3}\right)$. IRS revealed the composition of stones as hydroxyapatite, uric acid, cysteine, oxalic acid, and mixed type in $48,23,15$, five, and nine cases. The sensitivity and specificity of DECT for hydroxyapatite, uric acid, cysteine, oxalic acid, and mixed types were $89.6 \%$ and $88.5 \%, 82.6 \%$ and $97.5 \%, 86.7 \%$ and $96.5 \%, 80 \%$ and $98.9 \%$, and $88.9 \%$ and $98.9 \%$, respectively. On CT urography, a total of 35 had a high-grade and 65 had a low-grade obstruction, whereas DECT revealed high- and low-grade obstructions in 42 and 58 cases. The sensitivity and specificity of DECT for a highgrade obstruction were $94.3 \%$ and $86.2 \%$.

Received 04/06/2020

Review began 04/20/2020 Review ended 05/03/2020 Published 05/07/2020

๑) Copyright 2020 Singh et al. This is an open access article distributed under the terms of the Creative Commons Attribution License CC-BY 4.0., which permits unrestricted use, distribution, and reproduction in any medium, provided the original author and source are credited.

\section{Conclusions}

The findings of the study showed that DECT provides comprehensive information regarding the morphological, chemical, and anatomical characterization of ureteric stones.

Categories: Radiology, Urology, General Surgery

Keywords: chemical composition, dual energy ct, obstruction, ureteric calculi

\section{Introduction}

Urolithiasis is a common problem affecting $1 \%-5 \%$ of the population. It has a significant impact on the quality of life and leads to significant morbidity [1-2]. The worldwide prevalence ranges from $5 \%-9 \%$ in Europe, $7 \%-13 \%$ in North America, and $1 \%-5 \%$ in Asia [3].

The characterization of ureteric calculi and an understanding of their chemical composition is helpful from the point of view of their management. The study of urolithiasis is important not only because of the high recurrence rates and incidence but also because of the complications that arise from it like hydronephrosis leading to renal failure in the long run if the stone partially obstructs the urinary tract [4].

Imaging techniques, such as urography and ultrasonography (USG), are helpful in characterizing the morphology, size, number, and, to a certain extent, volume of the ureteric calculi. However, the estimation of the chemical composition of the calculus remains unknown. In recent years, dual-energy computed 
tomography (DECT) has emerged as a useful method not only for morphological and anatomical evaluation but also for chemical composition [4].

The advantage of DECT has been its ability to provide material-specific information that is unavailable on conventional single-energy CT without a significantly higher radiation dose. DECT is effective for the estimation of volume, character, and degree of obstruction in ureteric calculus [5].

The attenuation patterns and low- and high-energy levels are helpful in spectral separation and thus in understanding the chemical composition of the materials, as lighter materials show small differences in attenuation between low and high energy levels, whereas those heavier materials show larger differences in attenuation between low and high energy levels [6].

Chaytor et al. assessed the accuracy of DECT in characterizing urinary tract stone composition in patients presenting with renal colic and found it useful in the characterization of the renal stones [7]. Ege et al., in another study, assessed the incidence of the secondary signs associated with ureteral stones on unenhanced helical CT of patients with acute renal colic [8]. Out of the 110 patients, 91 had hydroureter, 88 had hydronephrosis, 65 had periureteric edema, and 57.2\% had unilateral renal enlargement. A total of 90 stones passed spontaneously and intervention was required in 21 cases. The authors found the CT scans useful in the assessment of the urinary obstruction.

In view of the promising role of DECT in stones characterization and association with urinary obstruction as seen in different studies, the present study was done at our tertiary care center to evaluate the volume of ureteric calculus, determine the composition of calculus materials, and measure the efficacy of DECT in the evaluation of perinephric edema to predict the degree of ureteral obstruction in patients with acute ureterolithiasis.

\section{Materials And Methods}

A prospective observations study was done in the Department of Radiodiagnosis over a period of one year. A total of 100 patients with ureteric calculus diagnosed on ultrasound/X-ray kidneys, ureters, and urinary bladder (KUB) in the age group of 18 to 70 years, who underwent invasive procedures, including ureterorenoscopy or who had spontaneous expulsion were enrolled in the study after taking informed consent. Approval from the Institutional Ethics Committee was obtained before starting the study. Pregnant women and the patients allergic to the contrast dye were excluded from the study.

The sample size was estimated based on the study by Ilyas M et al. who observed the sensitivity and specificity of DECT in differentiating a calcium oxalate from non-calcium oxalate calculus was $97.8 \%$ and $92.3 \%$, respectively [9]. Taking these values as a reference, the minimum required sample size with the desired precision of $10 \%, 80 \%$ power of study, and $5 \%$ level of significance was 72 patients. To reduce the margin of error, the total sample size taken was 100 .

The demographic information and presenting complaints of the patients were noted. A thorough history was taken and a physical examination was performed. DECT was performed using Siemens "SOMATOM-force (384 slices)" dual-source dual-energy CT machine with tin filter (Siemens AG, Munich, Germany) for an in vivo evaluation of volume and chemical composition. The patient was positioned supine on the CT table with an area of interest being the abdomen. A dual-energy scan was performed, which acquired the image from two tubes angled at $90^{\circ}$ - low energy $(80 \mathrm{eV})$ and high energy $(140 \mathrm{eV})$, respectively. The ratio of lowand high-energy attenuation was calculated (the DE ratio). Image acquisition and post-processing image interpretation were done per the technique described by Ilyas et al. [9]. For uric acid, cysteine, mixed, calcium oxalate, and hydroxyapatite calculi, the cut-off values of the DE ratio were taken as $<1.13,1.13-1.23$, 1.23-1.33, 1.33-1.53, and >1.53, respectively, and, accordingly, the chemical composition of the calculi was determined [9].

Excretory CT urogram was done to estimate the degree of obstruction as per the protocol of the modified version of the Society for Foetal Urology Hydronephrosis Grading System where no dilatation or local dilatation of the ureter and/or renal pelvis was categorized as low grade and calyceal dilatation along with ureter and renal pelvis dilatation was categorized as high-grade obstruction.

Post-surgical extraction or spontaneous expulsion, the size of the calculi was measured in the largest dimension. As some of the stones were fragmented during the procedure, the volume was assessed using the fluid displacement method (volumetric flask method), which was taken as the gold standard for comparison [10-11]. The chemical composition of the calculi was assessed through infrared spectroscopy (IRS).

DECT was performed on all the patients for an in vivo evaluation of volume and the chemical composition of the stones. The grade of obstruction was predicted on DECT subjectively, after the assessment of the perinephric edema in terms of strands of soft tissue attenuation in perinephric fat, perinephric fluid collection, and thickness of or collection of fluid along the renal fasciae [12-13]. 


\section{Cureus}

The efficacy of DECT for the determination of chemical composition and the prediction of the grade of obstruction was assessed in terms of sensitivity, specificity, positive predictive value (PPV) and negative predictive value (NPV), and accuracy, respectively.

\section{Statistical analysis}

Quantitative data were presented with the help of mean and standard deviation. A comparison among the study groups was done with the help of the unpaired t-test per the results of the normality test. Qualitative data were presented with the help of the frequency and percentage table. Association among the study groups was assessed with the help of the Fisher test, student t-test, and chi-square test. A p-value of less than 0.05 was taken as significant. Appropriate statistical software, including but not restricted to Microsoft Excel (Microsoft Corporation, Redmond, Washington) and SPSS ver. 20 (IBM Corp., Armonk, New York), was used for statistical analysis. Graphical representation was done in Microsoft Excel 2010.

\section{Results}

The age of patients ranged from 18 to 70 years, with a mean age of $45.6 \pm 11.7$ years. The majority (59\%) of patients were males. Eight patients reported a positive family history of urolithiasis. The post-extraction mean calculus size and volume were $9.45 \pm 7.13 \mathrm{~mm}$ and $65.1 \pm 77.61 \mathrm{~mm}^{3}$, respectively. The number of stones ranged from one to six, with a mean of $3.8 \pm 2.9$. Urography revealed a high-grade obstruction in $35 \%$ and a low-grade obstruction in 65\% cases. Post-extraction IRS revealed the chemical composition of stones as hydroxyapatite in the maximum number of cases (48\%) followed by uric acid (23\%), cysteine (15\%), and calcium oxalate (oxalic acid,5\%), respectively (Table 1).

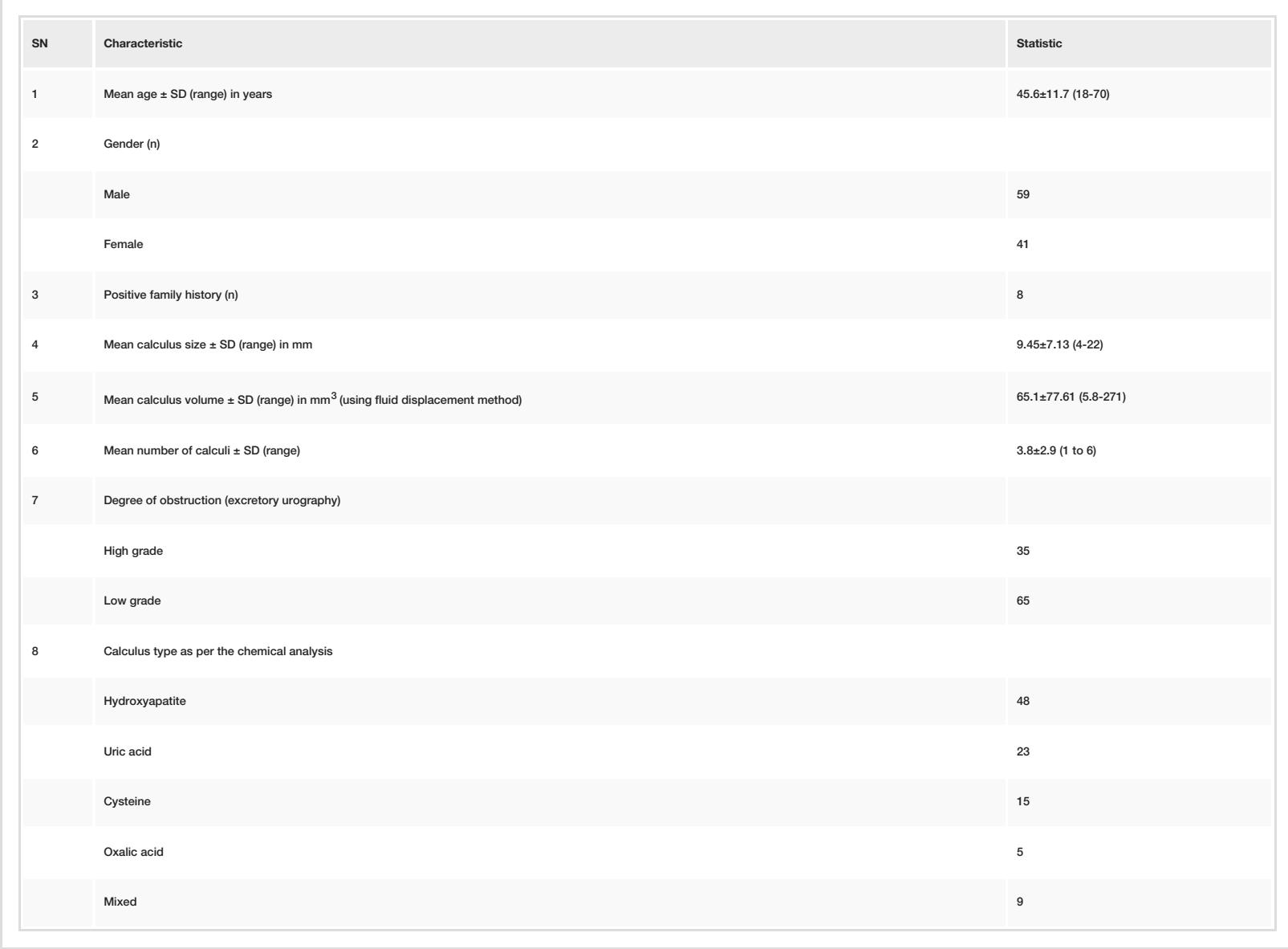

TABLE 1: Patient demographics, general characteristics, and chemical composition of ureteric calculi $(n=100)$

SD: standard deviation

On DECT, the mean CT attenuation values for low energy $(80 \mathrm{eV})$ and high energy $(140 \mathrm{eV})$ were $1271 \pm 178$ and $758 \pm 138 \mathrm{HU}$, respectively, for hydroxyapatite (mean DE ratio = 1.68), $534 \pm 62$ and $544 \pm 58 \mathrm{HU}$, respectively, for uric acid (mean DE ratio $=0.98$ ), $1110 \pm 146$ and $935 \pm 168 \mathrm{HU}$, respectively, for cysteine (mean $\mathrm{DE}$ ratio $=1.19$ ) and $853 \pm 284$ and $569 \pm 312 \mathrm{HU}$, respectively, for oxalic acid (mean DE ratio $=1.50)$ stones, 


\section{Cureus}

respectively. For cases with mixed stones, the attenuation values for low-energy and high-energy levels were $1203 \pm 327$ and $933 \pm 289$, respectively (mean DE ratio $=1.29$ ). The mean calculus volume as per the DECT measurement was $66.09 \pm 81.78 \mathrm{~mm}^{3}$. DECT diagnosed 42 cases as high-grade (dilatation of the ureter, renal pelvis, and calyces) and 58 cases as low-grade obstructions (local dilatation of the ureter and/or renal pelvis dilatation). On CT images, subjective assessment of the perinephric edema, fat stranding, and fluid collection suggested high-grade obstruction in $42 \%$ cases and low-grade obstruction in $58 \%$ cases (Table 2). Figures 1-3 show the DECT image of a right-sided ureteric stone characterized as hydroxyapatite stone along with perinephric and periureteric edema and fat stranding.

\begin{tabular}{|c|c|c|c|c|}
\hline Composition & No. of Cases & Mean \pm SD (HU) $80 \mathrm{eV}$ & MeantsD (HU) $140 \mathrm{eV}$ & DE Ratio \\
\hline Hydroxyapatite & 48 & $1271 \pm 178$ & 758土土138 & 1.68 \\
\hline Uric acid & 23 & $534 \pm 62$ & $544 \pm 58$ & 0.98 \\
\hline Cysteine & 15 & $1110 \pm 146$ & 935+168 & 1.19 \\
\hline Oxalic acid & 5 & $853 \pm 284$ & $569 \pm 312$ & 1.5 \\
\hline Mixed & 9 & $1203 \pm 327$ & $933 \pm 289$ & 1.29 \\
\hline \multicolumn{4}{|c|}{ Mean calculus volumeะSD (range) in $\mathrm{mm}^{3}$ on DECT } & $66.09 \pm 81.78(5.8-271)$ \\
\hline High grade & & & & 42 \\
\hline Low grade & & & & 58 \\
\hline
\end{tabular}

TABLE 2: DECT quantitative analysis for different types of calculi, volume, and degree of obstruction

DECT: dual-energy computed tomography; HU: Hounsfield unit; SD: standard deviation

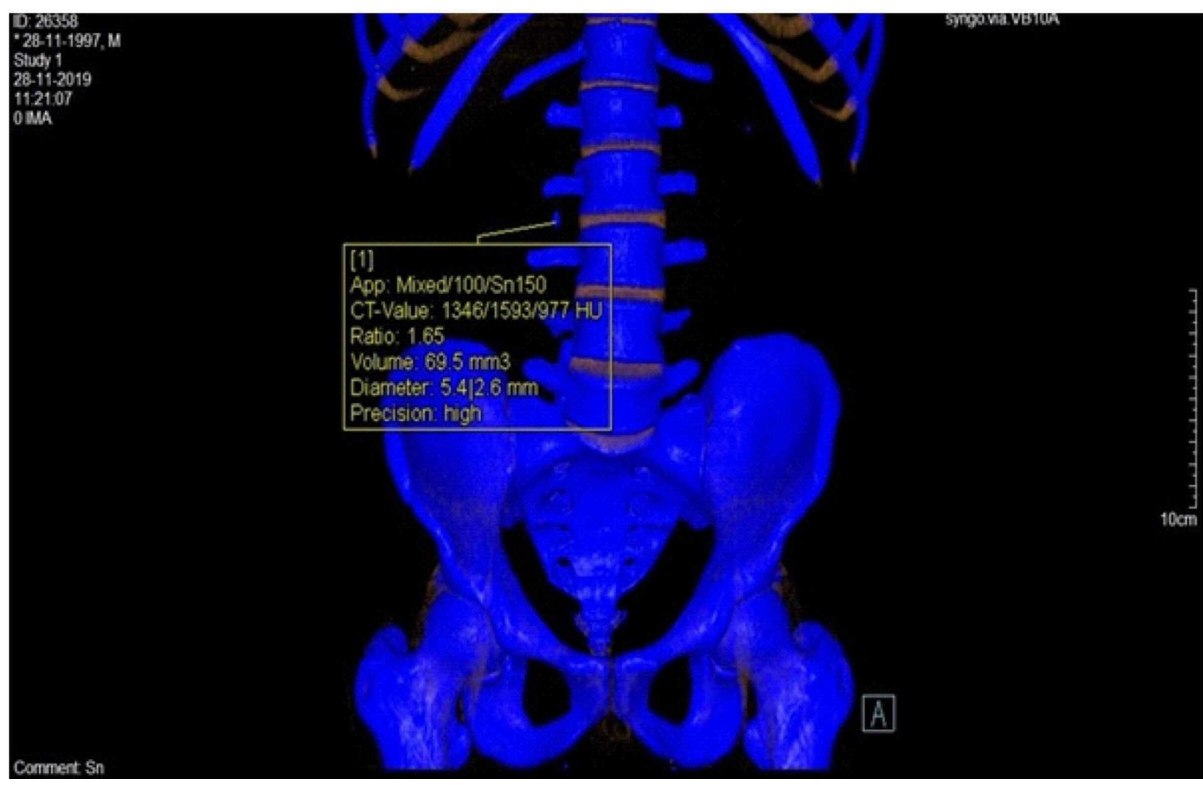

FIGURE 1: Noncontrast coronal dual-energy CT images with right-sided ureteric calculus

CT: computed tomography 


\section{Cureus}

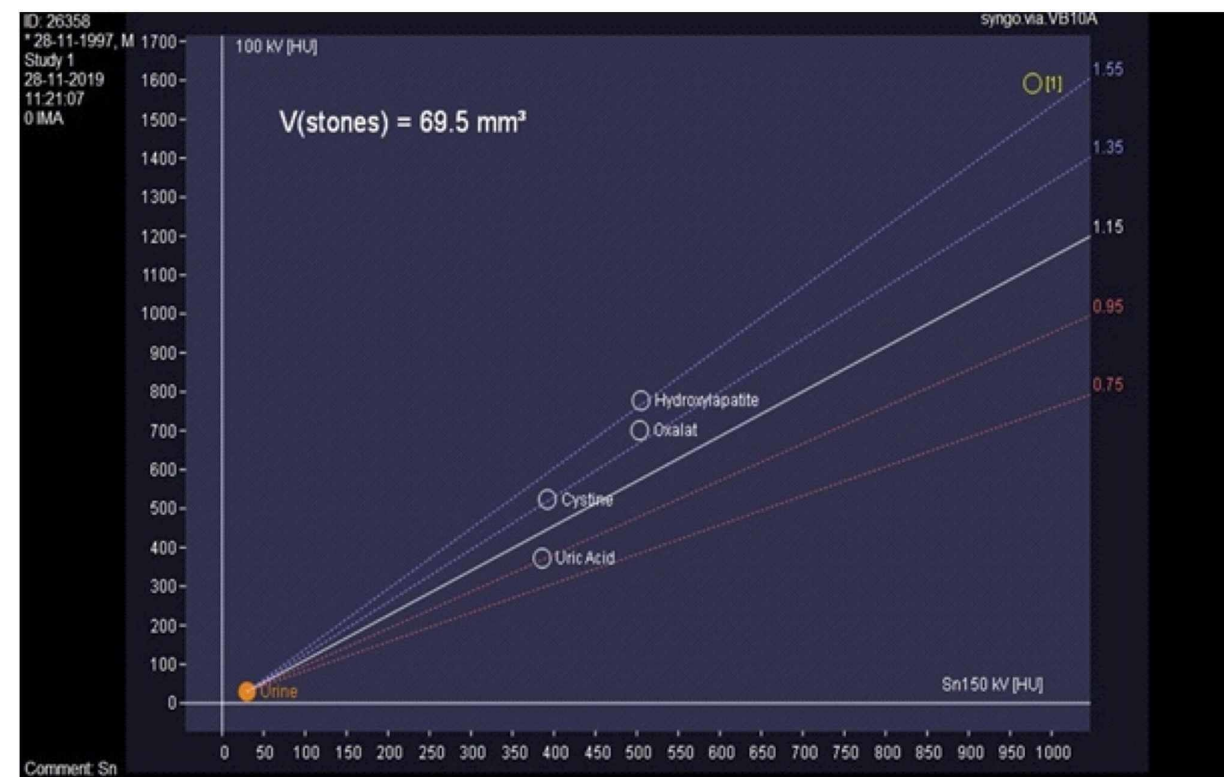

FIGURE 2: Graph showing the characterization of the right ureteric calculus (labeled 1) as hydroxyapatite stone

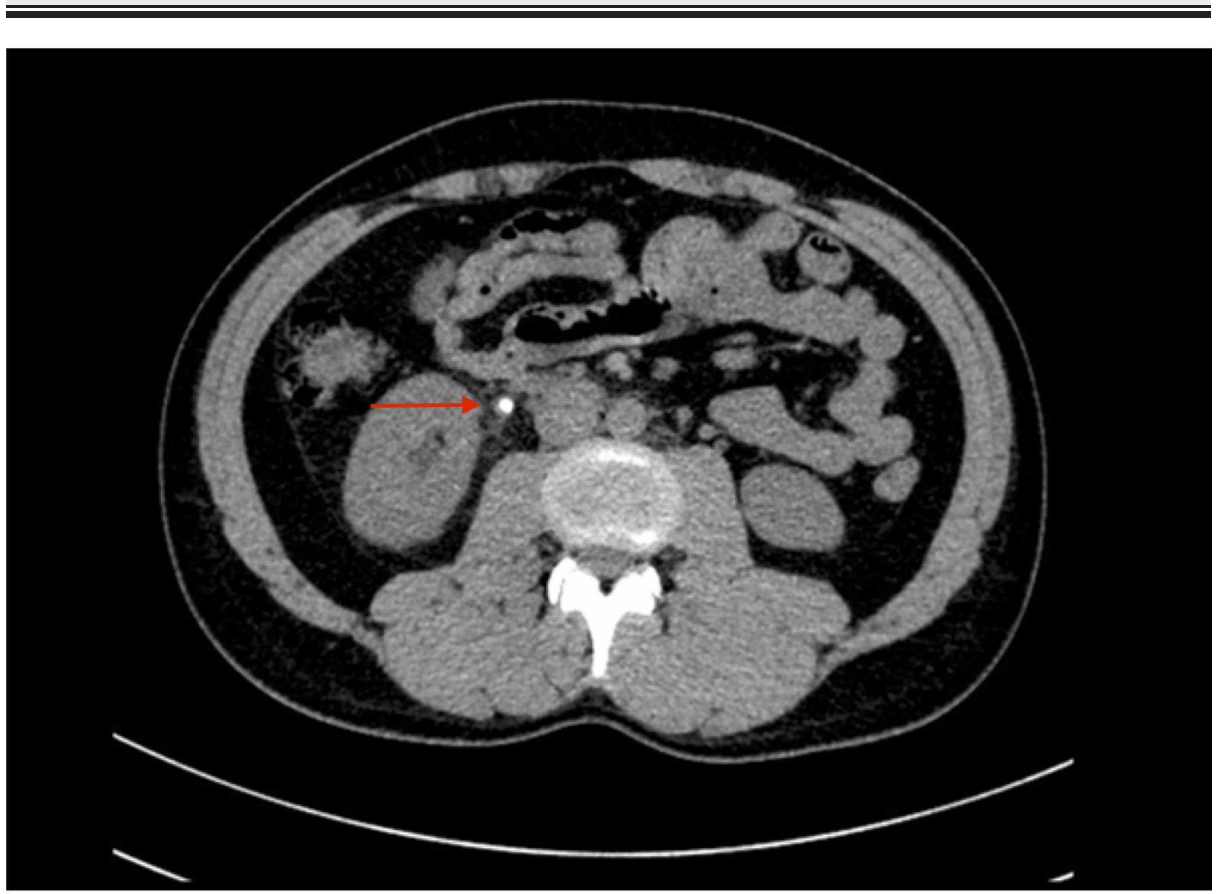

FIGURE 3: Axial non-contrast DECT image showing a calculus in the right proximal ureter with perinephric and periureteric fat stranding

DECT: dual-energy computed tomography

On DECT, a total of 49 calculi were diagnosed as hydroxyapatite, 19 as uric acid, 16 as cysteine, and five as oxalic acid. Nine cases were diagnosed as mixed type on DECT. On comparing the DECT findings with IRS findings, out of 49 cases diagnosed as hydroxyapatite, 43 (87.8\%) were true positive while six $(12.2 \%)$ were false positive (four uric acid and two cysteine calculi on IRS). On the other hand, out of 48 cases diagnosed as hydroxyapatite by IRS, only five (8.3\%) were false negative (three diagnosed as cysteine and one each diagnosed as mixed and oxalic acid respectively by DECT), thus 46 out of 52 (88.5\%) cases were true negative. Of the 21 cases diagnosed as uric acid by DECT, 19 (90.5\%) were true positive while two (9.5\%) were false positive (one each diagnosed as oxalic acid and mixed type by IRS). On the other hand, of the 23 cases diagnosed as uric acid by IRS and four (17.4\%) were false negative (diagnosed as hydroxyapatite by DECT), thus 75 out of 77 (97.4\%) were true negative. Out of 16 cases diagnosed as cysteine calculi by DECT, a 


\section{Cureus}

total of 13 (81.3\%) were true positive while three (18.7\%) were false positive (diagnosed as hydroxyapatite by IRS). Of the 15 cases diagnosed as cysteine calculi by IRS, two (13.3\%) were false negative (diagnosed as hydroxyapatite by DECT), thus a total of 82 out of 85 (96.5\%) were false negative. Both DECT and IRS diagnosed five cases as oxalic acid calculi. There was one false positive (diagnosed as uric acid by IRS) and one false negative (diagnosed as hydroxyapatite by DECT). Both DECT and IRS diagnosed nine cases each as mixed type. There was one false positive (diagnosed as hydroxyapatite by IRS) and one false negative (diagnosed as uric acid by DECT), respectively. Overall, there was an $87 \%$ agreement between IRS and DECT. On correlating the DECT-predicted chemical composition with IRS diagnosed chemical composition, the sensitivity, specificity, PPV, NPV, and accuracy of DECT for hydroxyapatite, uric acid, cysteine, oxalic acid, and mixed types were $89.6 \%, 88.5 \%, 87.8 \%, 92.2 \%$ and $89 \% ; 82.6 \%, 97.5 \%, 90.5 \%, 94.9 \%$ and $94 \%$; $86.7 \%$, 96.5\%, $81.3 \%$, $97.6 \%$ and $95 \%$; 80\%, $98.9 \%, 80 \%$, $98.9 \%$ and $98 \%$; and $88.9 \%, 98.9 \%, 88.9 \%, 98.9 \%$ and $98 \%$, respectively (Table 3).

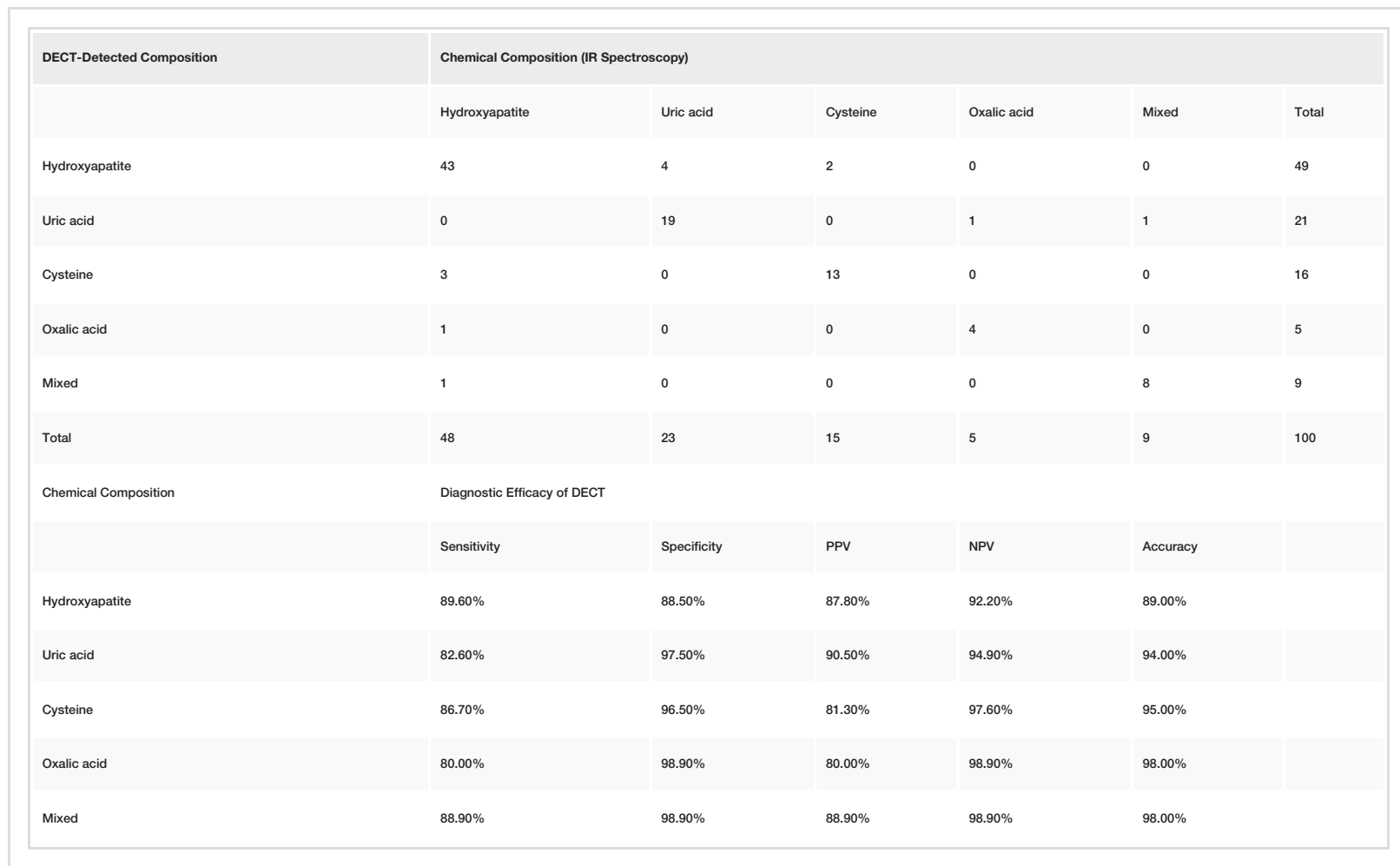

TABLE 3: Correlation between chemical composition and DECT-detected composition of ureteric calculi

DECT: dual-energy computed tomography; IR: infrared; NPV: negative predictive value; PPV: positive predictive value

Of the 35 cases diagnosed as high-grade obstruction, 33 (94.3\%) were true positive on DECT while 2 (5.7\%) were false negative whereas of the 65 cases diagnosed as low grade (not high grade) on urogram, 56 (86.2\%) were true negative and 9 (13.8\%) were false positive. Correspondingly, the sensitivity, specificity, PPV, NPV, and accuracy of DECT for the detection of urography-diagnosed high-grade obstruction was $94.3 \%, 86.2 \%$, $78.6 \%, 96.6 \%$, and $89 \%$ respectively (Table 4 ). 


\section{Cureus}

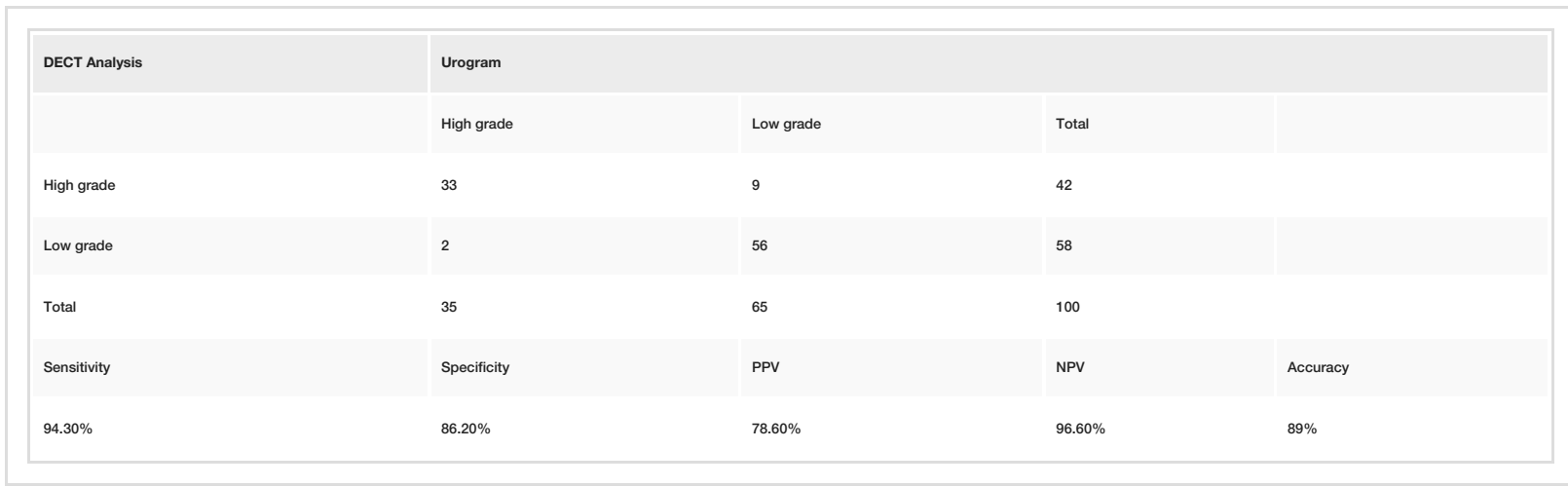

TABLE 4: Correlation between the degree of obstruction by urogram and DECT

DECT: dual-energy computed tomography; NPV: negative predictive value; PPV: positive predictive value

\section{Discussion}

The management of the ureteric stones depends upon the anatomical, morphological, and chemical characterization of the stones. It becomes imperative to know the features of the stone before operating, so as to provide the best management to the patient.

The present study found a high accuracy of DECT in the morphological assessment (volume of calculus) as well as the chemical composition. The mean calculus volume as per fluid displacement was $65.1 \pm 77.61 \mathrm{~mm}^{3}$, which did not differ significantly as compared to the DECT-measured calculus volume, which was $66.09 \pm 81.78 \mathrm{~mm}^{3}$. The mean difference between the DECT and in vitro assessment was $<1 \mathrm{~mm}^{3}$, showing high accuracy. The management of stone volume has a detrimental role in the choice of therapeutic approach. As per Narepalem et al., volume differences $>4.1 \mathrm{~mm}^{3}$ are clinically significant from the point of view of the selection of treatment options, however, DECT findings in the present study showed that the volume differences were not significant [11]. Similar observations were made by some previous studies too [12-14].

With respect to the degree of obstruction, the present study showed that DECT was $94.3 \%$ sensitive and $86.2 \%$ specific for the detection of high-grade obstructions as observed on excretory urogram. However, the accuracy of DECT was only $89 \%$, the reason for this could be a high false-positive rate (21.4\%) due to a subjective assessment of the degree of perinephric edema. However, one must understand that an excretory urogram is another imaging technique that has its own limitations in assessing the degree of obstruction and thus the correlations among both of them may further add to the false-positive rates. As compared to the index study, Boridy et al. in their study had shown the CT evaluation for the degree of obstruction was $100 \%$ sensitive and 100\% specific in view of intraoperative correlation [12]. These findings, in turn, suggest an additive role of DECT in the evaluation of the degree of obstruction.

As far as the chemical composition was concerned, we observed an $87 \%$ agreement between DECT and IRS. Among different types of calculi, maximum sensitivity was observed for hydroxyapatite (89.6\%) and mixed types (88.9\%) while maximum specificity was observed for mixed type and oxalic acid (98.9\% each). In fact, hydroxyapatite was the dominant type in the present study, seen in as many as $48 \%$ of cases by IRS and $49 \%$ of cases by DECT. This was followed by uric acid (23\%), cysteine (15\%), and oxalic acid (5\%) calculi while mixed stones were detected in $9 \%$ of cases. Compared to the present study, Erdogan et al. in their study found oxalic acid (42.9\%) and hydroxyapatite (36.5\%) as the dominant types on DECT while the in vitro analysis showed $22.9 \%$ as hydroxyapatite and $51.4 \%$ as oxalic acid calculi [15]. In another study, Ilyas et al. found oxalic acid as the dominant type seen in $78.4 \%$ of their cases [9]. Manglaviti et al. in their study did not diagnose hydroxyapatite in any of the cases and reported a dominance of oxalic acid (67.3\%) calculi [16]. In another study, Basha et al. described the chemical composition of ureteric calculi as calcium oxalate or phosphate (75.7\%) followed by uric acid (27.0\%) and cysteine stones (10.8\%), respectively [17]. In the present study, the proportion of hydroxyapatite and oxalic acid stones together was 53\% followed by uric acid (23\%) and cysteine (15\%), respectively. As far as differentiation between uric acid and non-uric acid stones is concerned, the present study is in concordance with the observations of Ilyas et al. who found 85\% of their sample to be non-uric acid and only $15 \%$ to be uric acid stones [9]. The reason for the difference in the chemical composition of stones in different studies could be attributable to the inclusion criteria used and the difference in dietary habits and ethnicity of the affected population. In addition, the different DE ratio cutoffs used for the characterization of ureteric stones among different studies may cause the difference in the results, necessitating the standardization of the DE ratios for in vivo stone characterization. 
in the present study (87\%), is in agreement with the observation made by Erdogan et al. who reported the accuracy of DECT to be $91.4 \%$ [15]. Manglaviti et al. in their study also reported an agreement in 45/49 (91.8\%) cases [16]. In the present study, the highest accuracy of stone detection was observed for oxalic acid and mixed types (98\%), whereas for hydroxyapatite, though the sensitivity was the maximum (89.6\%), the accuracy was minimum (89\%). This was owing to both high false positive $(n=6)$ as well as false negative $(n=5)$ rates. For uric acid and cysteine stones, the accuracy was $94 \%$ and $95 \%$, respectively. Ilyas et al. in their study observed $100 \%$ sensitivity and accuracy in the differentiation of uric acid from non-uric acid stones, whereas for the differentiation of oxalate from non-oxalate stones, the sensitivity and specificity values were $97.8 \%$ and $92.3 \%$, respectively [9]. Hydroxyapatite stones sometimes accompany infections, which could have played a role in affecting the attenuating values substantially [18]. The high accuracy of DECT in the detection of cysteine and uric acid stones has also been endorsed by Erdogan et al. in their study [15]. In the present study, there was no difficulty in diagnosing the mixed types, whereas Manglaviti et al. in their study found difficulty in the correct diagnosis of mixed types in four out of five mixed-type cases, probably owing to the fact that the stone size in their study had a diameter less than $1 \mathrm{~cm}$. In the present study, the average diameter of the stones was close to $1 \mathrm{~cm}(9.45 \mathrm{~mm})$ and those with mixed type either had multiple stones or had a diameter greater than $1 \mathrm{~cm}$, hence there was no such difficulty in their correct identification [16].

DECT has several important limitations in the characterization of urinary calculi. Accuracy in calculi characterization decreases when evaluating calculi $<3-5 \mathrm{~mm}$ because it is difficult to obtain accurate attenuation values [19].

Certain secondary signs aid in the diagnosis of ureteral stones on CT, including reliable signs, such as perinephric fat stranding, periureteral edema, hydroureter, and hydronephrosis, and less-consistent signs such as perinephric edema and lateral conal fascial thickening. The PPV and NPV of intra-renal collecting system dilatation and perinephric fat stranding in detecting ureterolithiasis near $98 \%$ and $91 \%$, respectively [8].

Such subjective additional signs were taken into account in the index study and thus based on CT images, perinephric edema was subjectively categorized into extensive and limited, which were suggestive of high-grade and low-grade obstruction, respectively. The study results showed that a high-grade obstruction was present in $42 \%$ cases while a low-grade obstruction was seen in $58 \%$ cases.

It was observed in the present study that agreement between the volume of calculus by volume fluid displacement and DECT assessments showed sensitivity $92.8 \%$, specificity $98.2 \%$, PPV $97.5 \%$, NPV 95\%, and accuracy $96 \%$. On the assessment of the volume of calculi, sensitivity was $92.8 \%$ and specificity was $98.2 \%$. The diagnostic accuracy was $96 \%$.

The index study findings are consistent with the study of Boridy IC et al., who reported that limited perinephric edema on CT images had a sensitivity of $88 \%$, a specificity of $100 \%$, PPV of $100 \%$, and NPV of $88 \%$ for the prediction that calculus was associated with a low-grade ureteral obstruction, whereas for highgrade ureteral obstruction, CT images had sensitivity 100\%, specificity 91\%, PPV 83\%, and NPV 100\% [12]. Similar findings were seen in the studies by Alice E et al. and Leng S et al. [13-14].

It must be kept in mind that the management strategy of the stones is greatly dependent upon the chemical composition of the stones. For instance, uric acid calculi can dissolve in the urine of higher $\mathrm{pH}$ and thus alkalization of the urine plays an effective role. On the other hand, another non-invasive management strategy, i.e., extracorporeal shock wave lithotripsy is less effective in the management of oxalic acid stones [20]. Thus, prior knowledge of the volume, degree of obstruction, and chemical composition of the stones can help in determining the route of management and could thus restrict the invasive procedures to the minimum level. The present study showed that DECT could prove to be a useful tool in the morphological, anatomical, as well as chemical characterization of urinary calculi and thus could be a useful tool in guiding the management strategy. It is a common perception that DECT scanning leads to increased ionizing radiation exposure as compared with conventional CT, which limits the wide implementation of DECT. However, it has been seen that radiation dose considerations with DECT are comparable to those achieved with conventional CT. Indeed, DECT exams for urolithiasis can be performed under $5 \mathrm{mSv}$ in ssDECT and dsDECT scanners.

One of the limitations of DECT is that it does not determine the functional status of the renal system, which is an important parameter for the management of ureteric stones. Secondly, the standard DE ratio cutoffs have not been established to characterize the ureteric stones that may affect the reported incidence of the chemical nature of the stones. In addition, we could not incorporate the intraoperative observations regarding the degree of obstruction to validate the overdetection of high grades of obstruction on DECT as compared to a urogram.

\section{Conclusions}


It can be concluded that DECT is a novel non-invasive imaging technique that provides comprehensive information and accurate prediction regarding the morphological, chemical, and anatomical characterization of ureteric stones. In addition, it showed high sensitivity and specificity for determining high-grade obstruction. The findings prompt the use of DECT as a potential tool for advanced treatment planning for urinary obstruction, thereby reducing the burden of surgical intervention.

\section{Additional Information \\ Disclosures}

Human subjects: Consent was obtained by all participants in this study. IEC Era's Lucknow Medical College and Hospital, Lucknow issued approval ELMC/R_cell/EC/2018/60. Animal subjects: All authors have confirmed that this study did not involve animal subjects or tissue. Conflicts of interest: In compliance with the ICMJE uniform disclosure form, all authors declare the following: Payment/services info: All authors have declared that no financial support was received from any organization for the submitted work. Financial relationships: All authors have declared that they have no financial relationships at present or within the previous three years with any organizations that might have an interest in the submitted work. Other relationships: All authors have declared that there are no other relationships or activities that could appear to have influenced the submitted work.

\section{References}

1. Holman CDJ, Wisniewski ZS, Semmens JB, Bass AJ: Changing treatments for primary urolithiasis Impact on services and renal preservation in 16,679 patients in Western Australia. BJU Int. 2002, 90:7-15. 10.1046/i.1464-410x.2002.02804.x

2. Singal RK, Denstedt JD: Contemporary management of ureteral stones . Urol Clin North Am. 1997, 24:59-70. 10.1016/s0094-0143(05)70354-2

3. Sorokin I, Mamoulakis C, Miyazawa K, Rodgers A, Talati J, Lotan Y: Epidemiology of stone disease across the world. World J Urol. 2017, 35:1301-1320. 10.1007/s00345-017-2008-6

4. Levine JA, Neitlich J, Verga M, Dalrymple N, Smith RC: Ureteral calculi in patients with flank pain: correlation of plain radiography with unenhanced helical CT. Radiology. 1997, 204:27-31. 10.1148/radiology.204.1.9205218

5. Demirel A, Suma S: The efficacy of non-contrast helical computed tomography in the prediction of urinary stone composition in vivo. J Int Med Res. 2003, 31:1-5. 10.1177/147323000303100101

6. Ramello A, Vitale C, Marangella M: Epidemiology of nephrolithiasis. J Nephrol. 2000, 13:45-50.

7. Chaytor RJ, Rajbabu K, Jones PA, McKnight L: Determining the composition of urinary tract calculi using stone-targeted dual-energy CT: evaluation of a low-dose scanning protocol in a clinical environment. Br J Radiol. 2016, 89:20160408. 10.1259/bjr.20160408

8. Ege G, Akman H, Kuzucu K, Yildiz S: Acute ureterolithiasis: incidence of secondary signs on unenhanced helical CT and influence on patient management. Clin Radiol. 2003, 58:990-994. 10.1016/s00099260(03)00294-0

9. Ilyas M, Dev G, Gupta A, Bhat TA, Sharma S: Dual-energy computed tomography: a reliable and established tool for In vivo differentiation of uric acid from nonuric acid renal Stones. Niger Postgrad Med J. 2018, 25:52-59. 10.4103/npmj.npmj 24 18

10. Demehri S, Kalra MK, Rybicki FJ, et al.: Quantification of urinary stone volume: attenuation threshold-based CT method--a technical note. Radiology. 2011, 258:915-922. 10.1148/radiol.10100333

11. Narepalem N, Sundaram CP, Boridy IC, Yan Y, Heiken JP, Clayman RV: Comparison of helical computerized tomography and plain radiography for estimating urinary stone size. J Urol. 2002, 167:1235-1238.

12. Boridy IC, Kawashima A, Goldman SM, Sandler CM: Acute ureterolithiasis: nonenhanced helical ct findings of perinephric edema for prediction of degree of ureteral obstruction. Radiology. 1999, 213:663-667. 10.1148/radiology.213.3.r99dc12663

13. Alice E. Huang AE, Juan C, Leng S, McCollough CH: Consistency of renal stone volume measurements across CT scanner model and reconstruction algorithm configurations. AJR Am J Roentgenol. 2017, 209:116121. 10.2214/AJR.16.16940

14. Leng S, Shiung M, Ai S, et al.: Feasibility of discriminating uric acid from non-uric acid renal stones using consecutive spatially registered low and high-energy scans obtained on a conventional CT scanner. AJR Am J Roentgenol. 2015, 204:92-97. 10.2214/AJR.13.11911

15. Erdogan $\mathrm{H}, \mathrm{Temizoz} \mathrm{O}$, Koplay M, Ozturk B: In vivo analysis of urinary stones with dual-energy computed tomography. J Comput Assist Tomogr. 2019, 43:214-219. 10.1097/RCT.0000000000000831

16. Manglaviti G, Tresoldi S, Guerrer CS, Leo GD, Montanari E, Sardarnelli F, Cornalba G: In Vivo Evaluation of the chemical composition of urinary stones using dual-energy CT. AJR Am J Roentgenol. 2011, 197:76-83. 10.2214/AJR.10.5217

17. Basha MAA, AlAzzazy MZ, Enaba MM: Diagnostic validity of dual-energy CT in determination of urolithiasis chemical composition: in vivo analysis. Egyptian J Rad Nucl Med. 2018, 49:499-508.

18. Park S: Medical management of urinary stone disease . Expert Opin Pharmacother. 2007, 8:1117-1125. 10.1517/14656566.8.8.1117

19. Jepperson MA, Cernigliaro JG, Sella D, Ibrahim E, Thiel DD, Leng S, Haley WE: Dual-energy CT for the evaluation of urinary calculi: image interpretation, pitfalls and stone mimics. Clin Radiol. 2013, 68:707-714. 10.1016/j.crad.2013.07.012

20. Liu Y, Qu M, Carter RE, et al.: Differentiating calcium oxalate and hydroxyapatite stones in vivo using dualenergy CT and urine supersaturation and pH values. Acad Radiol. 2013, 20:1521-1525.

10.1016/j.acra.2013.08.018 